\title{
Interactive comment on "Molecular fingerprinting of particulate organic matter as a new tool for its source apportionment: changes along a headwater drainage in coarse, medium and fine particles as a function of rainfalls" by Laurent Jeanneau et al.
}

\section{Laurent Jeanneau et al.}

laurent.jeanneau@univ-rennes1.fr

Received and published: 10 October 2017

Dear colleague, Many thanks for having taking the time to review our manuscript. I understand your idea of using this molecular fingerprinting method to differentiate between different litter types and soils. In this present paper, the sampling strategy was not design to investigate different litter types. However this method can be useful if there are important differences between plants in the catchment like gymnosperm ver- 
sus angiosperm. Such situations could be interesting to test this tool. Differentiating between soils is in the scope of this paper since forest, wetland and upland soils are used as potential end-members. Since the vegetation is quite homogenous in the riparian areas of this catchment, forest and wetland have a similar molecular fingerprint while upland is clearly differentiated. About your difficulty to read the manuscript, we will make the appropriate modifications in order to improve its understanding in the text, figure captions and table titles. About your question on the 71 variables used in the PCA. With this method, we analyzed 112 target compounds and their relative proportions (using relative distribution is necessary to compare organic rich and organic poor samples) are used as variables for the principal component analysis (PCA). Since some targets are correlated or appeared in low abundance, the set of variable was reduced to keep only the most representative, without correlation between variables. These are the 71 variables that will be identified on table S1. Once again, thank you for your time in reviewing this manuscript. Sincerely

On behalf of the coauthors, Laurent Jeanneau

Interactive comment on Biogeosciences Discuss., https://doi.org/10.5194/bg-2017-325, 2017. 